Document downloaded from:

http://hdl.handle.net/10251/123227

This paper must be cited as:

Martínez Abietar, AJ. (2018). Polarimetry enabled by nanophotonics. Science. 362(6416):750-751. https://doi.org/10.1126/science.aau7494

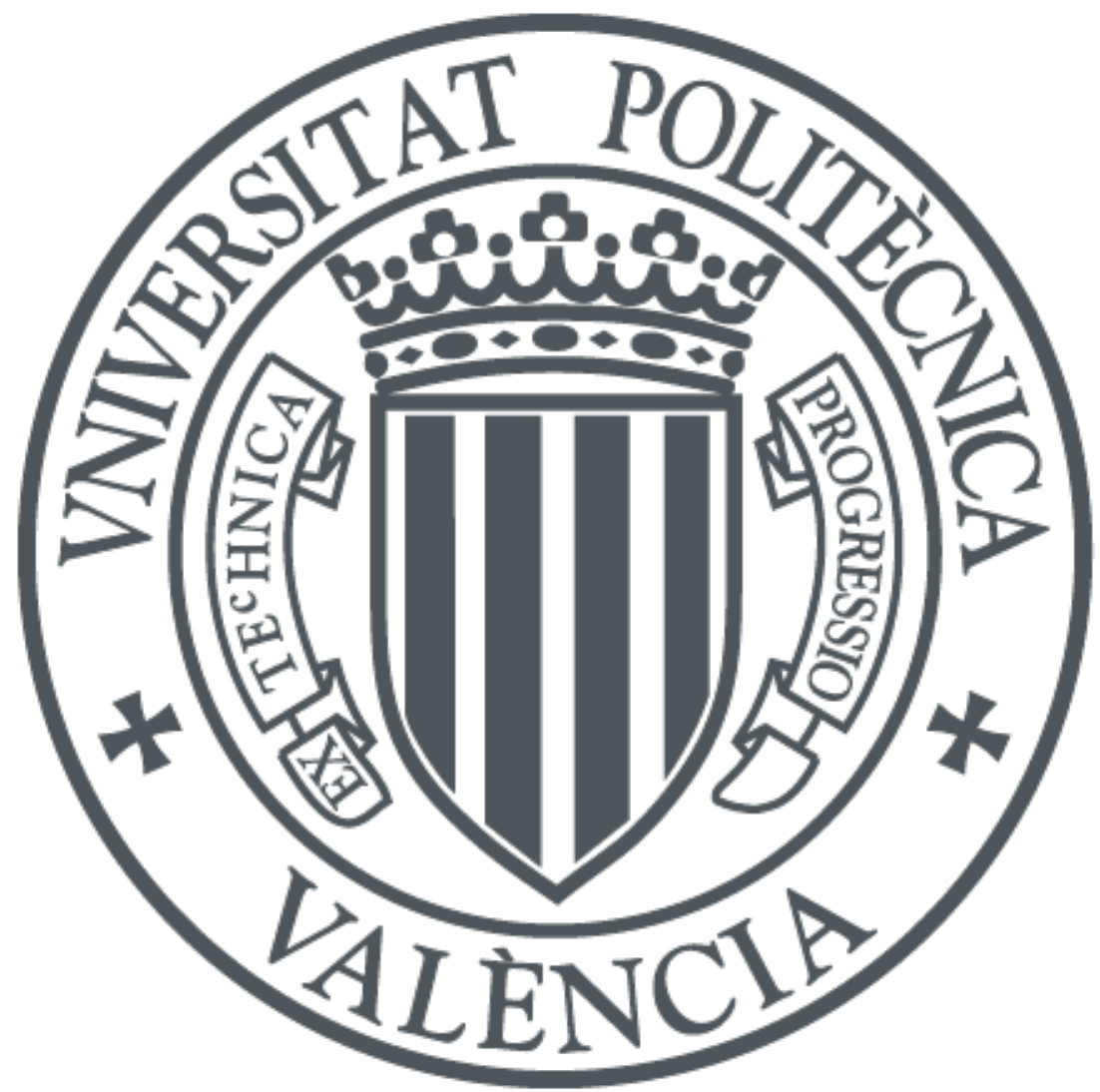

The final publication is available at

https://doi.org/10.1126/science.aau7494

Copyright American Association for the Advancement of Science (AAAS)

Additional Information 


\title{
Nanophotonics-enabled polarimetry
}

\author{
Alejandro Martínez \\ Nanophotonics Technology Center, Universitat Politècnica de València, Camino de Vera s/n, \\ Valencia, Spain
}

Light beams consist of oscillatory electric (and magnetic) fields having a certain amplitude, phase and frequency. In transverse waves, the state of polarization (SoP) characterizes how the electric field oscillates on the plane perpendicular to the propagation direction. Light-matter interaction strongly depends on the SoP, so its complete measurement is of paramount importance in a wide amount of disciplines, including chemistry, imaging, optical communications or astronomy, amongst others. However, measuring the SoP of a light beam, the main goal of polarimetry (1), is much trickier than knowing its intensity or frequency since it involves the simultaneous measurement of the four Stokes parameters, which even account for the case of unpolarized light. For decades, polarimeters have consisted of a combination of linear retarders, polarizers, and quarter-wave plates, which were able to get such four measurements by spatial or temporal splitting the incoming light beam (2). Such macroscopic polarimeters, widely used in many applications, are complex, bulky and expensive, and there have been little attempts for miniaturization with the notable exception of the fiber-grating polarimeter, highly useful in fiber optics (3).

Recent advances in nanoscience and nanotechnology have unveiled new ways to shrink polarimeters, which all the subsequent advantages that miniaturization and on-chip integration may bring (4). The underlying idea is that being the response of nanostructures to an incident light beam essentially polarization-dependent one may ultimately use such response to retrieve the SoP. For instance, a metasurface can be designed to scatter an incident light beam in different directions depending on its SoP (Fig. 1A), thus enabling to retrieve the Stokes parameters at a certain wavelength (5)-(9). Such scattering paths could be even parallel to the metasurface and therefore guided on the chip substrate, which could facilitate on-chip processing and detection (10). Still, light-matter interaction enabling the polarizationdependent response is distributed amongst all the elements of the array, so the device footprint is much larger than one square wavelength. Therefore, there is still room for further miniaturization.

Attaining truly nanoscale detection of the SoP requires polarization-dependent photonic resonances in individual metallic or dielectric nanostructures. For instance, one may think on using plasmonic nanostructures engineered in certain in-plane shapes so it can support optical hot-spots depending on the actual SoP of the illumination (Fig. 1B). By placing an absorbing semiconductor in the hot-spot regions, it will be possible to get output photocurrents proportional to the SoP (11). Even though the foot-print is much smaller than in the case of metasurface polarimeters, this approach still requires at least four nanostructures so that by measuring the photocurrent generated by each of them the SoP can be fully retrieved. Thus, this polarimeter would efficiently work for transverse light beams but would fail when measuring the SoP of complex, structured beams showing variation of the local polarization in the transverse plane (12).

Measuring the SoP at a single spatial point and in a single shot can be done by mixing both previous approaches: using polarization-dependent scattering from individual nanostructures. As a result of spin-orbit interaction (13), the direction of the scattered light paths will depend 
upon the incident polarization. Moreover, the scattering paths can be lithographically-defined waveguides supporting different modes that will carry all the information needed to retrieve the SoP (Fig. 1C) (14). Since in principle there is no limit for extremely downscaling the scatterer as long as it partly scatters the incoming beam, such polarimeter - which can be designed to operate optimally (15) - may be as small a single atom, though the efficiency of the system would also be subsequently reduced. Remarkably, spin-orbit interaction would also enable a full-vector description of the incident light, beyond the transverse picture (16).

By a suitable design (materials, shape, size) of the underlying nanostructures, the previous approaches can be used to build ultracompact polarimeters though the whole electromagnetic spectrum, even reaching the important THz regime. Moreover, they are extremely broadband: by calibrating the polarization scattering paths or absorption at each working wavelength, they can be employed for spectropolarimetry (7)-(8),(14). Notably, they may also be implemented using resonant dielectric nanostructures (17), besides avoiding the ohmic losses inherent to metals, also facilitates mass-manufacturing in silicon chips. Whilst the absorptive approach is highly appropriate to build a ending device for SOP measurement, the approaches based on scattering can operate in a non-destructive way with low insertion losses, being highly suitable for an in-line configuration. On-chip polarimeters should easily displace their bulk free-space counterparts because of the inherent advantages of integration, such low-cost, reliability, repeatability, or integration with electronics, to name a few. A number of applications would be immediately affected by the realization of such on-chip polarimeters. Low-cost inline polarimeters with low insertion losses operating at telecom wavelengths may be employed for real-time monitoring of the SoP in current and future optical communication networks employing polarization multiplexing schemes. Lightweight polarimetry chips operating in the visible could be easily integrated in satellites for measuring the magnetic activity on the Sun's surface (18). Spin-orbit polarimeters with ultimately reduced size may locally test the polarization of single photons in quantum systems and networks. Now that the fundamentals for on-chip nanoscale polarimeters have been settled down, it is time for making them a practical reality.

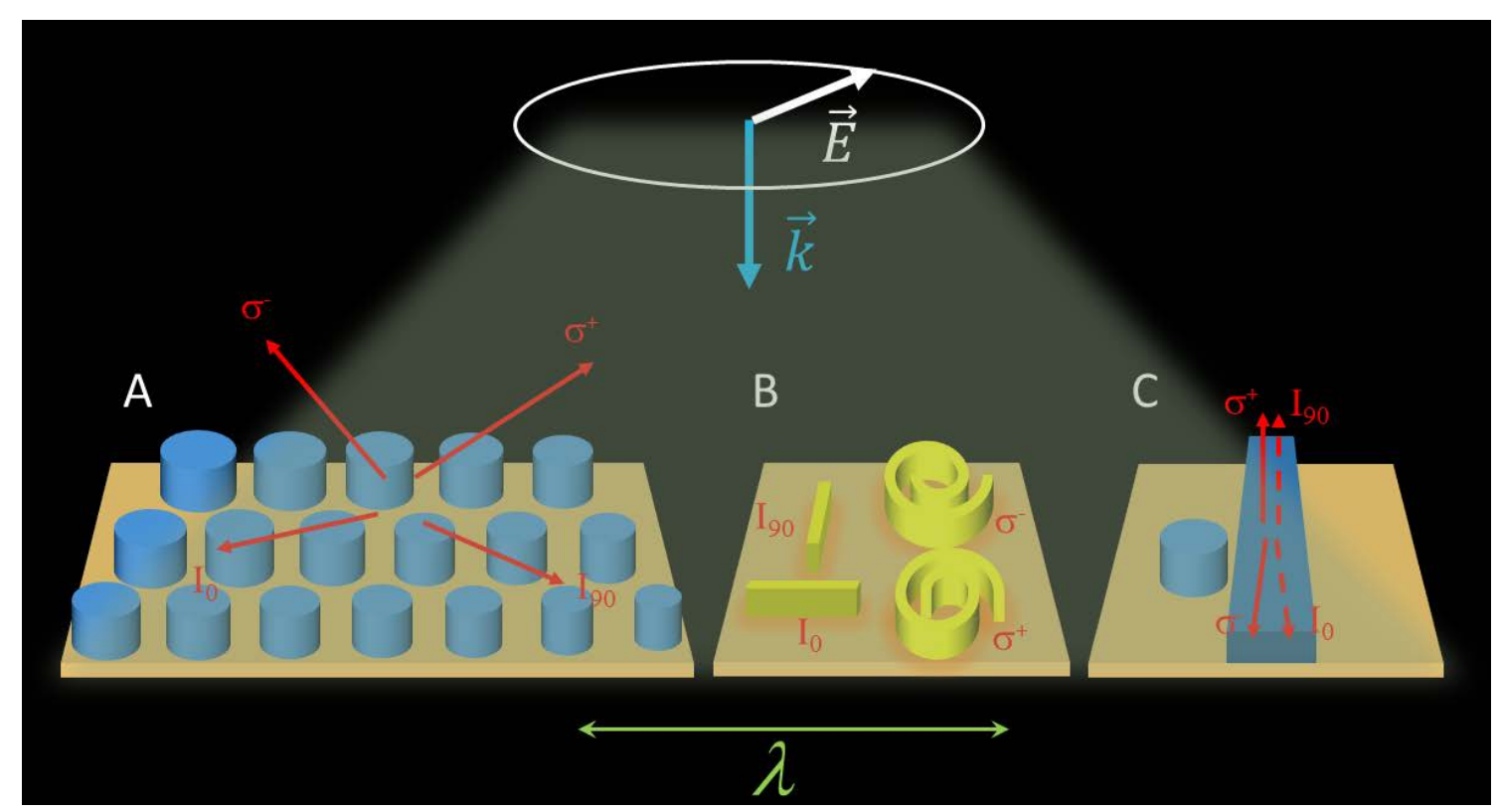

Figure 1. Nanoscale polarimetry. Transverse light (with a certain electric field E and wave vector $k$ ) illuminates a set of nanostructures able to determine its SoP. A. A metasurface consisting on a set of nanoantennas scatters different polarization states into well-defined 
spatial pathways. B. Plasmonic nanoantennas are shaped to preferably absorb light of a certain polarization. C. Spin-orbit interaction in a scatterer coupled to a waveguide scatters different input polarizations into different directions and modes of a waveguide. In all the cases, by measuring at least 4 outputs (corresponding to either scattered (A), absorbed (B) or guided (C) radiation) enables to retrieve the SoP. Possible polarization states are linearly polarized light forming either a $0^{\circ}\left(I_{0}\right)$ or a $90^{\circ}\left(I_{90}\right)$ with the horizontal axis, and right-handed $\left(\sigma^{*}\right)$ and lefthanded $(\sigma)$ circularly polarized light. Dielectric and metallic nanostructures are depicted in blue and yellow, respectively,

References:

(1) R. A. Chipman, Polarimetry. In Handbook of Optics; The McGraw-Hill Companies, Inc. (2010).

(2) R. M. A. Azzam, Stokes-vector and Mueller-matrix polarimetry, J. Opt. Soc. Am. A 33, 1396-1408 (2016)

(3) P. S. Westbrook, T. A. Strasser, and T. Erdogan, In-line polarimeter using blazed fiber gratings, IEEE Photon. Technol. Lett. 12, 1352-1354 (2000).

(4) A. Femius Koenderink, A. Alù, A. Polman, Nanophotonics: Shrinking light-based technology, Science 348, 516-521 (2015).

(5) A. Pors, M. G. Nielsen, and S. I. Bozhevolnyi, Plasmonic metagratings for simultaneous determination of Stokes parameters, Optica 2, 716-723 (2015).

(6) J. P. Balthasar Mueller, K. Leosson, and F. Capasso, Ultracompact metasurface in-line polarimeter, Optica 3, 42-47 (2016).

(7) W. T. Chen, P. Török, M. R. Foreman, C. Y. Liao, W.-Y. Tsai, P. R. Wu, and D. P. Tsai, Integrated plasmonic metasurfaces for spectropolarimetry, Nanotechnology 27, 224002 (2016).

(8) E. Maguid, I. Yulevich, D. Veksler, V. Kleiner, M. L. Brongersma, E. Hasman, Photonic spin controlled multifunctional shared-aperture antenna array, Science 352, 1202 1206 (2016).

(9) E. B. Magnusson, J. P. Balthasar Mueller, M. Juhl, C. Mendoza, and K. Leosson, Neural Polarimeter and Wavemeter, ACS Photonics, Article ASAP (2018).

(10)A. Pors and S. I. Bozhevolnyi, Waveguide Metacouplers for In-Plane Polarimetry, Appl. Phys. Rev. 5, 064015 (2016)

(11)F. Afshinmanesh, J. S. White, W. Cai, and M. L. Brongersma, Measurement of the polarization state of light using an integrated plasmonic polarimeter, Nanophotonics 1, 125-129 (2012).

(12)T. Bauer, P. Banzer, E. Karimi, S. Orlov, A. Rubano, L. Marrucci, E. Santamato, R.W. Boyd, and G. Leuchs, Observation of optical polarization Möbius strips, Science 347, 964 (2015).

(13)K. Y. Bliokh, F. J. Rodríguez-Fortuño, F. Nori, and A. V. Zayats, Spin-orbit interactions of light. Nat. Photonics 9, 796-808 (2015).

(14)A. Espinosa-Soria, F. J. Rodríguez-Fortuño, A. Griol, and A. Martínez, On-chip optimal Stokes nanopolarimetry based on spin-orbit interaction of light, Nano Lett. 17, 31393144 (2017).

(15)M. R. Foreman, A. Favaro, A. Aiello, Optimal Frames for Polarization State Reconstruction. Phys. Rev. Lett. 115, 263901 (2015).

(16)T. Bauer, S. Orlov, U. Peschel, P. Banzer, G. Leuchs, Nanointerferometric amplitude and phase reconstruction of tightly focused vector beams, Nat. Photonics 8, 23-27 (2014). 
(17)A. I. Kuznetsov, A. E. Miroshnichenko, M. L. Brongersma, Y. S. Kivshar, and B. Luk'yanchuk, Optically resonant dielectric nanostructures, Science 354, aag2472 (2016).

(18)J. O. Stenflo, Solar Magnetic Fields (Kluwer, 1994). 